\title{
Rursus
}

Russus

Poiétique, réception et réécriture des textes antiques

$6 \mid 2011$

Relire, récrire, prolonger. Adaptations gréco-latines

\section{Horace ou le refus de la poésie grecque courtisane}

\section{Dominique Voisin}

\section{(2) OpenEdition \\ Journals}

Édition électronique

URL : http://journals.openedition.org/rursus/465

DOI : $10.4000 /$ rursus. 465

ISSN : 1951-669X

Éditeur

Université Nice-Sophia Antipolis

\section{Référence électronique}

Dominique Voisin, «Horace ou le refus de la poésie grecque courtisane », Rursus [En ligne], 6 | 2011, mis en ligne le 28 janvier 2011, consulté le 20 avril 2019. URL : http://journals.openedition.org/ rursus/465; DOI : 10.4000/rursus.465

Ce document a été généré automatiquement le 20 avril 2019

Rursus 


\title{
Horace ou le refus de la poésie grecque courtisane
}

\author{
Dominique Voisin
}

1 Les poètes latins, de manière générale, et Horace, en particulier, semblent s'intéresser fort peu aux lettrés étrangers qui font partie de la domus de leur puissant dédicataire ${ }^{1}$. Je voudrais apporter deux brèves analyses à l'appui de cette présentation volontairement romanisée de la vie littéraire et artistique faite par le poète : une étude comparative de deux poèmes adressées à Caius Sallustius Crispus, l'un de Crinagoras, l'autre d'Horace et, de manière plus générale, une évaluation critique des mentions que fait Horace des personnalités célébrés par les poètes grecs contemporains, Crinagoras bien sûr, mais également Antipater de Thessalonique et Apollonide de Nicée.

\section{Caius Sallustius Crispus, Horace, 0. 2, 2 et Crinagoras, Anth. PI., 40}

2 Selon Tacite ${ }^{2}$, Caius Sallustius Crispus eut en commun avec Mécène de mener une vie élégante et raffinée, de refuser la carrière sénatoriale, tout en jouant un rôle politique important en tant que conseiller d'Auguste d'abord, de Tibère ensuite. Ce chevalier, partisan d'Antoine avant Actium, devint l'un des amis les plus intimes du Prince, tout de suite après Mécène, le premier après la mort de celui-ci ${ }^{3}$. Petit-neveu et fils adoptif de l'historien Salluste, il pouvait avoir hérité de ce dernier sinon des aptitudes, du moins des goûts littéraires. F. Della Corte voit dans Sallustius Crispus un autre Mécène, c'est-à-dire un autre protecteur et ami des poètes ${ }^{4}$ ? L'universitaire italien s'appuie tout particulièrement sur le témoignage de Tacite. Mais cet historien donne fort peu d'informations sur les goûts littéraires des hauts personnages qu'il décrit, y compris des Princes; je crois plutôt qu'il a avant tout dans l'esprit, lorsqu'il compare Sallustius à Mécène, le rôle politique du ministre souterrain, rôle effectivement rempli par Sallustius, notamment auprès de Tibère, lors du meurtre d'Agrippa Postumus. En revanche, Suétone est très attentif aux intérêts littéraires des Grands ${ }^{5}$; or, il ne mentionne jamais Sallustius Crispus. ${ }^{6}$ 
3 Sans ambiguïté, Crinagoras célèbre la générosité et l'opulence de Sallustius, celle que contemplait la foule admirative devant les fameux horti hérités de l'historien, celle que commémore Tacite dans sa notice nécrologique :

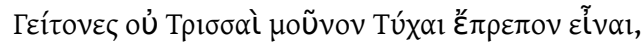

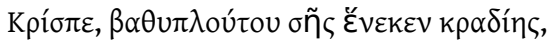

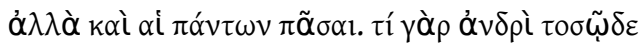

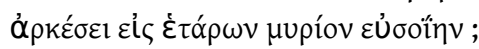

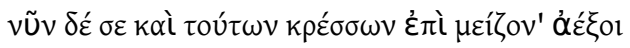

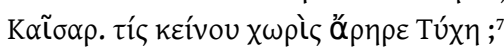

4 La fortune de Sallustius est moins louée que sa magnificence envers ses amis ; l'emploi du

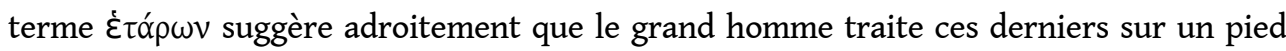
d'égalité mais n'indique aucunement que ces amis sont des lettrés ou des littérateurs; or, la tradition grecque encomiastique mentionne le plus souvent l'avantage particulier apporté par une contribution littéraire, soulignant ainsi le goût du dédicataire pour les lettres ${ }^{8}$. Une exagération plaisante, qu'accompagne le double sens de Túxๆ, permet au poète de reprendre un lieu commun moral tout en louant le donateur (les biens de fortune sont passagers ; la générosité de Sallustius est telle qu'il risque de se ruiner) et de subordonner l'éloge de l'ami du Prince à la louange du Prince lui-même : seul César est maître de la Fortune et seule sa munificence envers Salluste pourrait permettre à ce dernier de continuer à se montrer généreux. Les quatre derniers vers suggèrent que Sallustius joue un rôle d'intermédiaire entre le Prince et les bénéficiaires de ses largesses, ce que l'Ode d'Horace ne corrobore pas. Etant donné que les épigrammes de Crinagoras que nous avons conservées, concernant les personnalités marquantes de cette époque, célèbrent uniquement des personnes liées à Auguste ${ }^{9}$ ou Auguste lui-même ${ }^{10}$, nous pouvons considérer que Crinagoras applique le raisonnement du rhéteur étranger répondant à Auguste qui proposait de lui faire connaître un autre Grand: "Tant que le soleil brille, je n'allume pas de lampe $»^{11}$. La pointe de l'épigramme grecque, en vue de laquelle celle-ci est construite, concerne César. Sallustius Crispus, parce qu'il est un exemplum connu de générosité, transformé lui-même en bénéficiaire, sert de faire-valoir à la munificence impériale. Le poème de Crinagoras révèle moins le rôle d'intermédiaire joué par Sallustius dans le patronage des poètes (rien ne révèle l'intérêt pour les lettres dans cette épigramme) que l'évolution pyramidale de la société, tout éloge d'un personnage important étant subordonné à l'éloge du Prince.

Voilà déjà une première différence avec l'ode d'Horace. Que Sallustius Crispus soit un ami d'Auguste n'explique pas, en effet, forcément la dédicace du poète lyrique; d'ailleurs, aucune mention n'est faite d'Auguste. Horace, peut-être ex-antonien lui-même, célèbre dans le livre 2 des Odes d'autres antoniens, repentis (tel Q. Dellius en $0.2,3$ ) ou non (tel Pollion en $0.2,1)$. Horace met en scène avec une ironie plaisante Dellius, le voltigeur des guerres civiles, pour illustrer la leçon philosophique de l'égalité d'âme. Ne pourrait-il se servir également avec humour de l'opulent Sallustius pour dénoncer «deux maux contraires et également funestes, l'amour du luxe et l'amour de l'argent $»^{12}$ et opposer à "l'éclat périssable des richesses» la vertu qui "assure la gloire et l'immortalité »" autres topoi de la morale antique? Tous les contemporains lettrés d'Horace devaient se souvenir que le grand-oncle de notre Sallustius avait tout particulièrement utilisé ces lieux communs afin d'expliquer les causes de la dégénérescence de l'État romain. Il était donc amusant d'appliquer à l'héritier, rallié à la politique augustéenne, les leçons de l'historien libéré des périls d'une « ambition mauvaise »" 
6 Le premier quatrain enferme Sallustius entre deux extrêmes, l'auaritia, qui est plaisamment évoquée par l'hypallage de la terre avare (les lecteurs d'Horace connaissaient les mines de cuivre du val d'Aoste exploitées par Sallustius ${ }^{15}$ ) et la vaine gloire de la luxuria, suggérée par la valeur hyperbolique du verbe splendere. Au centre du quatrain, Sallustius est d'abord désigné, de manière surprenante pour tous ceux qui connaissent son faste, par le vocatif inimice lamnae : le grand homme serait-il transformé en Sage méprisant les richesses? Non pas, car la connotation philosophique de l'adjectif contraste avec le très concret et légèrement péjoratif lamnae ${ }^{16}$ et alerte le lecteur : Sallustius est l'ennemi du métal brut ; il doit donc être l'ami du métal qui brille, c'est-àdire de l'argent monnayé ; mais, pour éviter que l'ami d'Auguste ne soit transformé en prodigue, le participe temperato indique par avance que le dédicataire d'Horace sait l'art philosophique de la modération (à moins qu'il ne s'agisse tout simplement d'un simple calcul: Salluste sait être généreux sans pour autant se ruiner, ce que contredit l'épigramme de Crinagoras). La place du dédicataire, au centre du quatrain, ne figureraitelle pas alors la mediocritas aurea chère au poète? Il n'est point sûr, vu le témoignage de Tacite, que les lecteurs d'Horace eussent choisi cette interprétation.

7 La seconde strophe présente un exemplum de gloire véritable, celui de Proculeius, qui n'a pas hésité à partager toute sa fortune avec ses frères ${ }^{17}$ et révèle, par contraste, ce qu'a d'ambigu la présentation de Sallustius. Ce n'est donc pas l'argent, même bien géré, qui confère un éclat immortel, mais le sacrifice de cet argent au nom de la pietas.

8 Les trois dernières strophes opposent l'esprit d'avidité, qui appauvrit l'homme le plus fortuné, à la véritable sagesse ${ }^{18}$, sans en donner d'exemplum contemporain. Mais les deux derniers vers, qui décrivent le regard méprisant du Sage, même devant des monceaux d'or, ferment élégamment la composition circulaire du poème et rappellent en écho l'attitude de Sallustius à la première strophe. Celui-ci méprisait les trésors cachés sous la terre seulement parce qu'ils étaient inexploités. De plus, la litote inimicus... nisi soulignait que le petit neveu de l'historien n'avait pas l'indifférence du Sage pour l'argent. Le poète ne fait-il pas ici comme la «Vertu » ? En « désaccord avec la foule », et conformément aux réflexions morales développées par le grand-oncle du richissime homme d'État, il « retranche » ce dernier « du nombre des heureux ». Horace a sans doute eu connaissance

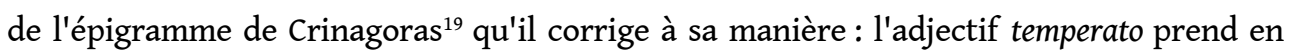
effet le contre-pied de la générosité sans borne décrite par l'épigrammatiste grec; au thème de la Fortune se substitue celui de la Sagesse. A l'hommage courtisan adressé à l'ami du prince, le lyrique latin préfère un portrait ambigu ${ }^{20}$, destiné moins à Sallustius qu'à des lecteurs lettrés, amusés par la comparaison entre l'historien moraliste pastiché par le poète et le politique opportuniste.

\section{Les Pisons, Horace et les épigrammatistes grecs}

9 Il serait tentant de voir dans Pison le Pontife, né en 48, consul en 15 av. J.C. ${ }^{21}$, le destinataire de l'épître d'Horace ${ }^{22}$. En effet, ce Pison a hérité des goûts philhelléniques de son père, Piso Caesoninus, le protecteur du philosophe épicurien Philodème. Lui-même protège des écrivains grecs, tels les épigrammatistes Antipater de Thessalonique et Apollonide de Nicée. C'est donc manifestement un lettré. Nous avons conservé six poèmes d'Antipater en l'honneur de Pison et de sa carrière ${ }^{23}$ et un poème d'Apollonide en l'honneur d'un Gaius, fils de Lucius, pour sa première barbe ${ }^{24}$. 

conception épicurienne de la poésie divertissement défendue par Philodème ${ }^{25}$. Un autre noble lettré, de six ans plus jeune, partageait les goûts de Caesoninus pour la poésie grecque légère : il s'agit de Tibère ${ }^{26}$, dont Pison fut le conseiller intime ${ }^{27}$ et sous le règne duquel il exerça les fonctions de praefectus urbi jusqu'à sa mort, en 32 ap. J.C. ${ }^{28}$. Or, Tibère et le poète Horace ne s'appréciaient guère ${ }^{29}$.

11 Si Horace a dédié l'Art poétique à Pison le Pontife, nous devons considérer que le poète, encore une fois, a fait preuve d'un humour déconcertant vis-à-vis de son dédicataire. Cet Art poétique n'est-il pas une réécriture d'un traité du néo-académicien Néoptolème, traité

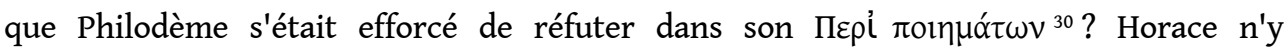
condamne-t-il pas l'esthétique des cantores Euphorionis, telle celle de Furius Bibaculus ? ${ }^{31}$ Ne propose-t-il pas également de convertir la poésie privée, raffinée, que l'élite avait choisie pour meubler aristocratiquement ses loisirs, en " une parole officielle qui sorte des cercles et s'impose sur le théâtre au peuple " ? ${ }^{32}$ Dans cette perspective, Pison le Pontife et ses fils auraient été choisis parce qu'ils incarnaient les partisans d'une poésie désengagée de l'otium qu'il fallait convertir à la nouvelle poésie selon Horace, celle qui allie le raffinement de la forme et l'expression des valeurs civiques ${ }^{33}$.

Cependant, aucun qualificatif précis de cette épître ne désigne les Pisons comme des lettrés. Les rares formules qui les mentionnent les associent sous le terme générique de Pisones (vers 6 et 235) et soulignent, dans les fils, l'héritage des vertus paternelles (ce qui est conforme à la tradition aristocratique). Elles exaltent l'origine mythique de cette noble famille descendant de Numa Pompilius, le roi qui institua le droit, les lois et les bonnes moeurs à Rome. Elles distinguent enfin, dans l'âné des fils, les bienfaits de l'éducation paternelle et leur conséquence, les qualités personnelles de jugement, voire la sagesse socratique ${ }^{34}$, qu'en digne fils de son père il commence à manifester. Ces trois Pisons incarnent donc l'archétype de la famille noble romaine. Tacite pourrait permettre de les identifier.

Les Annales présentent, en effet, le portrait de trois Pisons, un père et ses deux fils qui incarnent la ferocia aristocratique républicaine: Cneus Piso, consul en 23 av. J.C., et ses deux fils, Cneus, questeur en 19 av. J.C., consul en 7 av. J.C. et Lucius, l'Augure, consul en 1 av. J.C. ${ }^{35}$ Selon l'historien, Cneus Calpurnius Piso, le fils aîné, est un survivant, face à Tibère, de l'ancienne liberté républicaine ${ }^{36}$. L'historien ne le sépare pas de son père ${ }^{37}$. Son frère, Lucius, se souviendra toute sa vie d'avoir été le fils du compagnon de Brutus et de Cassius $^{38}$. consulat de leur(s) dédicataire(s), nous devons dater l'Art poétique de 23 av. J.C. L'aîné des fils est âgé alors de 20 ans (il commence donc sa vie publique sous la conduite de son père, conformément aux vers 366-367); le plus jeune est âgé de 14 ans (ce qui explique l'absence de référence personnelle).

Quelle est la fonction littéraire d'une dédicace destinée à des personnages qui, apparemment, ne sont pas passionnés de littérature? En tant que famille aristocratique nouvellement ralliée ${ }^{39}$, les Pisons sont les destinataires judicieux d'une «Défense et Illustration de la littérature latine », telle que la conçoit Horace ${ }^{40}$. Ils pourraient en effet être tentés, notamment le jeune $\mathrm{Cn}$. Pison, de s'illustrer par l'écriture, mode pernicieuse que le poète dénonce aux vers 382-384 et 416-421: un riche et noble amateur ne peut être un écrivain véritable. En revanche, le véritable poète est un personnage sacré, un 
interprète des dieux (vers 391), fondateur de villes et législateur avisé (vers 394-399) : c'est à lui que revient la gloire et la vraie noblesse (vers 400-401 et 406-407).

Les conseils exagérés donnés au jeune Pison, dans le cas où celui-ci s'aviserait d'écrire, soulignent l'abîme qui sépare l'artiste inspiré de l'amateur bien né, même quand ce dernier fait preuve de goût et de sagesse philosophique. Le tribunal imaginaire devant lequel il devra soumettre sa production poétique illustre, plus particulièrement, la nouvelle conception horatienne de l'art dramatique qui vise à réconcilier théâtralité et textualité, dimension publique et dimension artistique. Le jeune homme devra d'abord obtenir l'approbation du spécialiste du théâtre romain, Sp. Maecius Tarpa : celui-ci est le garant de l'efficacité spectaculaire de la pièce, sans plus ${ }^{41}$. Ensuite, il prendra l'avis de son père : celui-ci, descendant du législateur Pompilius, vérifiera si son fils a bien appris «ce qu'il doit à sa patrie, à ses amis, de quelle affection il faut aimer un père, un frère, un hôte, quel est le devoir d'un sénateur ou d'un juge, le rôle du général envoyé à la guerre ", s'il "sait infailliblement donner à chaque personnage les traits qui lui conviennent" (vers 312-316). Enfin, il entendra la sentence d'Horace, le maître en poésie ${ }^{42}$ : ce dernier veillera sans doute à la perfection d'un poème que de multiples ratures auront élagué, poli pendant dix ans (vers 293-294), où l'agréable se mêlera à l'utile et assurera le renom de l'écrivain (vers 344-346).

Si aucun intérêt littéraire ne liait le poète à ses dédicataires ${ }^{43}$, en revanche, le père avait combattu à Philippes aux côtés d'Horace, ainsi que L. Calpurnius Bibulus (S. 1, 10, 86), Pompeius $(0.2,7)$, et $\mathrm{L}$. Sestius Quirinalis, le dédicataire de l'Ode 1 , 4, dont le consulat de 23, contemporain de celui de Pison, permet de dater la publication des trois premiers livres des Odes $^{44}$. Cet homo nouus, ancien préteur de Brutus, qui rendait un culte à la mémoire des «Libérateurs", avait été, comme Horace, un des tribuns militaires du tyrannicide ; il partageait également le goût du poète pour la philosophie épicurienne, si l'on en croit le poème qui lui est dédié; ce dernier ne contient aucune allusion politique ${ }^{45}$, célèbre les plaisirs du printemps et décrit l'approche de la mort. Peut-être Sestius écrivait-il lui aussi des vers érotiques appréciés (vers 19-20). Le choix malicieux d'un poème de symposion pour célébrer un consul qui apparaît, comme Thaliarque, en arbiter bibendi (vers 18) est significatif. Auguste vient de renoncer à revêtir, année après année, la magistrature suprême; lui succède, de manière éclatante, un ancien adversaire, comme pour attester la totale liberté du jeu des institutions républicaines. Loin de célébrer cet honneur politique par une ode civique, Horace laisse entendre que le riche Sestius demeure un homme privé, jouissant des plaisirs de l'otium (c'est-à-dire sans aucun pouvoir politique).

Ainsi, la double dédicace à $\mathrm{L}$. Sestius et à son collègue Pison invite le lecteur à rapprocher les trois premiers livres des Odes et l'Art poétique. Les vers 13-14 de l'ode rencontrent un écho lyrique, peu approprié à une épître didactique, dans les vers 63-68 de l'Art Poétique : nouvel indice de la coïncidence chronologique des deux textes. Autre écho pessimiste, celui qui apparaît dans $0.2,10,9-12$. Or, cette ode est vraisemblablement dédiée à Varro Murena, le consul condamné et remplacé par Cneus Pison. En outre, l'Art poétique (vers 438-444) fait l'éloge du critique modèle récemment disparu, Quintilius Varus célébré en 0. 1, 24. Attaquant les partisans des Anciens et des cantores Euphorionis, défendant le labor limae, la fonction morale et civique du poète et sa véritable noblesse, l'Art poétique est l' Impromptu de Versailles du lyrique qui répond ainsi aux détracteurs des Odes. ${ }^{46}$ Dans le choix de ses dédicataires, Horace ne vise pas un intérêt immédiat courtisan ; il s'adresse avant tout à ses lecteurs, sub specie aeternitatis. 


\section{L'image de la famille impériale, Horace et les épigrammatistes grecs}

Les grandes familles aristocratiques de la République ont toujours su célébrer leurs ancêtres et exploiter leurs liens familiaux. Comme la famille du Prince devient la première famille aristocratique de l'État, il est naturel qu'une confusion s'instaure entre les célébrations privées et les cérémonies publiques ${ }^{47}$. Horace n'a guère participé à cette mise en scène publique de la famille du Prince.

La femmes de la famille impériale, réduites au nombre de deux, n'ont droit qu'à une seule apparition dans toute l'oeuvre d'Horace $(0.3,14,5-8)$. Le poète, jouant le rôle du héraut ordonnateur d'une cérémonie publique, célèbre le retour triomphal d'Auguste revenu d'Espagne en 24 av. J.C. Il place Livie et Octavie en tête du cortège des mères dont les fils et les filles viennent d'être sauvés par les victoires du Prince. Leur double statut (elles sont liées personnellement au chef victorieux et elles sont des matrones) leur permet d'avoir une fonction d'intercesseur et de souligner les liens privilégiés qui existent entre Auguste et la plèbe romaine qu'il protège paternellement. Cette présentation est pleinement en accord avec l'orthodoxie augustéenne. Conformément au rôle public adopté par le poète, aucun détail ne suggère un lien personnel entre Horace d'une part, Octavie et Livie de l'autre.

21 Mais Horace n'en reste pas là. Il passe ensuite à un autre registre et transforme cette ode triomphale en chanson à boire. Il rappelle alors son impétuosité amoureuse passée, à l'époque du consulat de Plancus. Ce qui lui offre une transition élégante avec l'ode suivante : au contraire d'un Horace résigné à la vieillesse, la vieille épouse d'un certain Ibycus, au lieu de filer la laine comme il convient à son âge, se livre encore impudiquement aux plaisirs de l'amour. Ainsi, $0.3,14$ et 3,15 invitent à comparer le passé et le présent. "Que faisait donc Livie, image idéale de la matrone quae lanam facit, à l'époque du consulat de Plancus?», pourrait se demander un lecteur facétieux et précédemment intrigué par l'épithète unico du vers 5 de l'Ode 3, $14^{48}$. En effet, la deuxième strophe de cette ode offre une symétrie curieuse entre la dénomination d'Octavie, soror clari ducis, et celle de Livie, unico... mulier marito. Mais le sens d'unicus fait problème. Considérer que l'expression unico marito est une périphrase faisant de Livie une uniuira serait rendre Horace coupable d'un mensonge criant et peu habile ${ }^{49}$. Doit-on choisir alors le sens d' «exceptionnel »? Le prince serait loué pour ses glorieuses qualités de chef, ainsi que dans la formule soror clari ducis. En ce cas, la symétrie des deux expressions unico marito et clari ducis suggère également que le Prince brille par ses mérites singuliers en tant que mari et le lecteur est de nouveau ramené au passé et aux deux divorces successifs d'Octave le triumvir. Nous devons craindre en outre qu'un ancien lecteur de Catulle, inspiré par le voisinage de dux et d'unicus, ne se rappelle fâcheusement l'interpellation ironique du vers 11 du Carmen 29 qui fustige Jules César, imperator unice. Ainsi, l'image idéalisée de la famille impériale dans les quatre premières strophes de 0.3 , 14 est rétrospectivement écornée par la remise en cause irrévérencieuse de la dernière strophe et de 0.3, 15.

En revanche, des épigrammatistes font l'éloge des femmes de la famille, tel le romain Domitius Marsus qui semble être le précurseur d'une "poésie romaine de cour et de circonstance $\aleph^{50}$, ou le grec Crinagoras ${ }^{51}$. Horace, d'ailleurs, ne mentionne pas plus l'épigrammatiste Domitius Marsus que Crinagoras. Les grands poètes latins, en général, n'ont guère été inspirés par ce type de commémoration. Pourtant, de nombreux mariages 
et anniversaires eussent été de faciles prétextes à des célébrations ${ }^{52}$ et les Carmina catulliens en proposaient des exemples réussis ${ }^{53}$.

Si de telles contributions poétiques semblent prévisibles dans un contexte historique aussi favorable, en revanche, une nouveauté du régime liée à la glorification de la famille princière est bien plus difficilement acceptable par la nobilitas: c'est la question de la transmission des pouvoirs publics à l'intérieur de la domus privée du Prince ${ }^{54}$. Jusqu'en 4 ap. J.C.55, Auguste a soigneusement évité de prononcer des termes signifiant qu'il confierait les pouvoirs de l'État à ses descendants ${ }^{56}$, tout en orchestrant publiquement, quoique implicitement, dès 29 , cette transmission ${ }^{57}$. Si les poètes, conformément aux volontés d'Auguste, n'ont pas traité ce thème, du moins pouvaient-ils, s'ils devaient obéir à des contraintes de propagande, l'illustrer indirectement, comme le faisait Auguste. Or, aucun des grands poètes n'a célébré Marcellus de son vivant. Chez Horace apparaît seulement, dans $0.1,12,45-46$, M. Claudius Marcellus: le vainqueur de Syracuse, récompensé par les spolia opima, cinq fois consul, figure dans un catalogue de héros républicains comparable à celui de Virgile $(E n .6,824-853)$ et de Manilius $(1,788)$. Pour trouver des éloges de Marcellus écrits de son vivant, nous devons nous tourner encore une fois vers les épigrammatistes grecs, Crinagoras en l'occurrence ${ }^{58}$.

Le quatrième livre des Odes d'Horace exalte, certes, maintes personnalités de la famille impériale : Paulus Fabius Maximus $(0.4,1)$, Iullus Antonius (0.4, 2), Drusus $(0.4,4)$ et Tibère $(0.4,14)$. J'ai déjà étudié les limites de ces commémorations dans le cas des deux derniers personnages ${ }^{59}$. Des intérêts littéraires communs expliquent et inspirent, bien plus que des motifs politiques ${ }^{60}$, les dédicaces à Fabius Maximus et à Iullus Antonius. $\mathrm{Si}$ deux vers de Properce $(4,6,81-82)$ esquissent le rôle militaire futur de Gaius et Lucius, les petits-fils d'Auguste, Horace les ignore totalement.

C'est donc dans les épigrammes, grecques en particulier, que la famille princière reçoit, proportionnellement, les hommages les plus attentifs et les plus précoces. De fait, les vers épigrammatiques apportent une réponse directe et immédiate aux événements typiques de la vie publique ou privée ; ils sont donc un genre qui convient parfaitement à la poésie de cour. Or, ces vers occasionnels relèvent, chez les Romains, de la poésie privée et légère propre aux amateurs ${ }^{61}$. Ils ne sont guère aptes à consacrer la gloire de leurs auteurs et sont dépourvus de la dimension civique qui convient lorsqu'on célèbre une famille qui incarne les valeurs de l'État romain. Par ailleurs, les grands poètes sont conscients de construire une oeuvre digne de l'éternité. Même lorsque cette oeuvre est composée de pièces distinctes, le soin porté à l'arrangement et à la succession des poèmes prouve que les écrivains s'efforcent de fondre tous les composants de leurs ouvrages dans leur projet poétique. Horace recrée «son » Auguste, pacificateur et législateur, paterfamilias, garant de l'otium du poète lyrique tout autant que des valeurs morales du satiriste et de l'épistolier philosophe. Quels personnages faire jouer à Livie ou à Octavie dans une oeuvre lyrique ? L'unique rôle qui leur convienne est celui de matrone romaine! C'est celui que Properce fait tenir à Cornélie dans une épitaphe, seule forme élégiaque appropriée à ce caractère. Quant aux enfants de la famille impériale, si souvent représentés dans les arts plastiques, les poètes latins, héritiers d'une tradition littéraire qui avait négligé jusque-là les enfants en général ${ }^{62}$, n'ont pas jugé bon d'innover à leur propos ${ }^{63}$. Ainsi, conformément à la tradition littéraire romaine et fidèle à son projet poétique original, Horace s'est interdit d'exploiter le thème, si cher à Auguste, de la famille impériale, se distinguant, là encore, des poètes grecs courtisans. 


\section{BIBLIOGRAPHIE}

ALEXANDER W.H., « Nullus argento color (Odes 2, 2, 1-4) », TAPhA, 74, 1943, pp. 192-201.

ALFONSI L., « Verba seniorum. Di pagani e cristiani », Bolletino di Studi Latini, 6, 1976, pp. 288-294.

ARGENTIERI, L., Gli epigrammi degli Antipatri, Bari, Levante, 2003.

BEAUJEU J., « L'Enfant sans nom de la 4 ${ }^{\mathrm{e}}$ Bucolique », REL, 60, 1982, pp. 186-215.

BOWERSOCK G. W., Augustus and the Greek World, Oxford, Clarendon Pr., 1965.

CALDER W.M., « Irony in Horace Carm. 2, 2 », CPh, 56, 1961, pp. 175-178.

CAMERON A. D. E., « Crinagoras and the elder Julia : Anth. 6.345 », LCM, 1980, 5, pp. 129-130

CHAUMONT M.L., « Echos de la campagne de Tibère en Arménie, (20 av. J.C.), dans une épigramme

de Crinagoras (Anth. 9, 430) », AC, 61, 1992, pp. 178-189.

CITTI V. « Una eco delle Georgiche in un epigramma di Apollonide (AP. 9, 244) » in UGLIONE R. (dir.) Atti del convegno nazionale di studio su Virgilio, Torino 1-2 maggio 1982, Torino, Edizioni dell'Orso 1984, «Collana Atti dei convegni della Delegazione torinese dell'Associazione italiana di cultura classica » pp. 165-169.

Coco, L. « Crinagora di Mitilene epigrammista », C \& S, 1993, 125, pp. 73-77.

CRAWFORD, M.H., « Greek Intellectuals and the Roman Aristocracy », in GARNSEY P.D.A. \& WHITTAKER C.R. (dir.), Imperialism in the Ancient World, Cambridge, 1978, « Class. Stud. », pp. 193-207.

DELlA CORTE F. (dir.), Q. Orazio Flacco, Le Opere I, Le Odi, Il Carme seculare, Gli Epodi, Roma, I.P.Z.S. Libreria dello Stato,1991.

DESCHAMPS L., « Salluste inspirateur d'Horace », Orphea Voce, 3, 1990, pp. 45-58.

DUPONT F. et NÉRAUDAU J.P., « Marcellus dans le chant 6 de l'Enéide », REL, 48, 1970, pp. 259-279.

DUPONT F., L'Acteur-Roi, Paris, Les Belles Lettres, 1985, « Coll. Realia ».

DURET L., « Dans l'ombre des plus grands », ANRW, 2, 30, 3, 1983, pp. 1447-1560.

FRISCHER B., Shiftings Paradigms : New Approaches to Horace's Ars Poetica, Atlanta, Scholars Pr., 1991.

GASCOU J., Suétone historien, Paris, de Boccard, 1984, « Coll. de l'École franç. de Rome ».

GOAR R. J., « Horace, Velleius Paterculus and Tiberius Caesar », Latomus, 35, 1976, pp. 43-54.

GONZALES J., « The first Oath pro salute Augusti found in Betica », ZPE, 72, 1988, pp. 113-127.

GRIMAL P., « Horace et la question du théâtre à Rome ", in GRIMAL P., Rome. La littérature et l'histoire, Paris, de Boccard, 1986, «Coll. de l'École franç. de Rome », pp. 67-80.

GRIMAL P., « Les vingt-trois premiers vers de l'Art poétique d'Horace », Vita Latina, 104, 1986, pp. 2-8.

GRIMAL P., Essai sur l'Art poétique d'Horace, Paris, Soc. d'éd. d'enseign. sup., 1968. 
GROSS W.H., Ways and Roundabout Ways in the Propaganda of an Unpopular Ideology, inWINKES R. (dir.), The Age of Augustus, Providence, Louvain-la-Neuve, 1985, « Publ. d'histoire de l'art \& d'archéol. de l'Univ. cathol. de Louvain, 44 », « Archaelogia Transatlantica, 5 », pp. 29-50.

Holloway R.R., "Who's Who in the Ara Pacis? », in Studi e Materiali (Studi in onore di Achille Adriani ), 6, Roma, 1984, pp. 625-628.

Kellum B., " Scuptural Programs and Propaganda in Augustan Rome: The Temple of Apollo in the Palatine », in winKES R. (dir.), The Age of Augustus, Providence, Louvain-la-Neuve, 1985, « Publ. d'histoire de l'art \& d'archéol. de l'Univ. cathol. de Louvain, 44 », « Archaelogia Transatlantica, $5 »$, pp. 169-176.

KLEINER D.E.E., " Private Portraiture in The Age of Augustus », in WINKEs R. (dir.), The Age of Augustus, Providence, Louvain-la-Neuve, 1985, « Publ. d'histoire de l'art \& d'archéol. de l'Univ. cathol. de Louvain, 44 », « Archaelogia Transatlantica, 5 », pp. 107-136.

LAUGIER J. L., « Notes sur la date de l'Art poétique d'Horace », Orphea Voce, 3, 1990, pp. 159-166.

MICHEL A., « Horace lecteur d'Horace : le livre 2 des Epîtres », in CHEVALLIER R. (dir.),

« Présenced'Horace », Caesarodunum, 23 bis, 1988, pp. 199-209.

Millar F. et SEGAL E. (dir.), Caesar Augustus : Seven aspects, Oxford, Clarendon Pr., 1984.

NÉRAUDAU J.P., La Jeunesse dans la littérature et les institutions de la Rome républicaine, Paris, Les Belles Lettres, 1979, « Coll. d'Ét. anc.».

RAAFLAUB K. \& TOHER M. (dir.), Between Republic and Empire. Interpretations of Augustus and His Principate, Berkeley, Univ. of California Pr.,1990.

SYME R., The Augustan Aristocracy, Oxford, Oxford, Clarendon Pr., 1986.

SYME R., Roman Papers, Oxford, Oxford, Clarendon Pr., 1984, p. 1089.

TREGGIARI S., Intellectuals, Poets and their Patrons in the firstcentury B.C., Echos du monde classique, Classical News and Views, 1, 1977, pp. 24-29.

VoISIN D., « Le pouvoir et ses écritures : fonction politique et poétique des hommages horatiens à Tibère et ses amis » in LOPEZ D. (dir.), Le Pouvoir et ses écritures, Bordeaux, PUB, 2010 (à paraître).

VoISIN D., « De l'ignorance réciproque des poètes latins et des poètes grecs contemporains » in BIRAUD M. et BOSC C. (dir.), Aux sources du métissage culturel : aspects scientifiques, linguistiques et artistiques dans l'Antiquité, Actes des Journées de la CNARELA, Nice, 27 et 28 Octobre 2008, publication électronique.

WHITE P., « Amicitia and the Profession of poetry in early imperial Rome », JRS, 68, 1978, pp. 74-92. WHITE P., « Positions for Poets in Early imperial Rome », in GOLD B. K. (dir.), Literary and artistic Patronage in Ancient Rome, Austin, Univ. of Texas Pr., 1982, pp. 50-66.

WILLIAMS G., " Phase in Political patronage of Literature in Rome », in GOLD B. K. (dir.), Literary and artistic Patronage in Ancient Rome, Austin, Univ. of Texas Pr., 1982, pp. 3-27.

WISEMAN T.P., «Pete nobiles amicos : Poets and Patrons in Late Republican Rome », dans GolD B. K. (dir.), Literary and artistic Patronage in Ancient Rome, Austin, Univ. of Texas Pr., 1982, pp. 28-49. woodman T. et WEST D. (dir.), Poetry and Politics in the Age of Augustus, Cambridge, Cambridge Univ. Pr.,1984. 


\section{NOTES}

1. Cette indifférence est réciproque: aucun lettré grec de cette période ne mentionne les grands auteurs latins contemporains, à l'exception de l'ami de Denys, Caecilius de Caleacté, qui compare (GELL., 19, 9, 7) Cicéron à Démosthène (CRAWFORD 1974 : 196-197; voISIN 2008).

2. TAC., An. 1, 6; 2, 40; 3, 30.

3. Son fils adoptif épousa une Domitia, petite-nièce d'Auguste (symE 1986 : 300).

4. DELLA CORTE 1991 : pp. 77-78.

5. GASCOU 1984 : pp. 668-673.

6.

7. CRINAGORAS A. Pl. 40: «Trois fortunes pour voisines, non, ce n'est pas assez, Crispus, pour un coeur aussi généreux que le tien: il te faudrait toutes celles du monde! Pour une âme pareille, quels biens suffiraient à combler ses amis? Puisse aujourd'hui, plus fort que toutes ces fortunes, César t'élever plus haut encore! Est-il sans lui de fortune assurée? » (sauf mention contraire, les éditions de référence sont celles de la CUF).

8. PD., P. 1.92-94; BACCHYL., Epinic. 3, 13; THCR., Charites 29-30, 90-98.

9. Les épigrammes 6, 161 et 9, 545 de l'Anthologie Palatine sont destinées à Marcellus; 6, 244 et 9, 239 concernent Antonia; 6, 229 désigne Lucius, peut-être L. Iulius Caesar; 9, 430 contient une allusion au fleuve Araxe, au bonnet de feutre des Arméniens et à la brebis de race " agarrique »; le poème évoque sans doute la campagne de Tibère en Arménie (CHAUMONT 1992 : 178-189).

10. Anth. 9, 419 et $562 ; 9,516$ fait allusion aux Ligures.

11. SÉN., Contr. 9, 5, 21.

12. SAL., C. 5,8 .

13. SAL., C. $1,4$.

14. SAL., C. 5, 2. Sur Horace lecteur de Salluste, DESCHAMPS $1990:$ 47-58.

15. PLIN., H.N. 34, 3.

16. ov., F. 1, 208: leuis lammina.

17. Porphyrion commente ainsi la générosité de Proculeius: Proculeius eques Romanus amicus Augusti carissimae pietatis erga fratres suos Scipionem et Murenam fuit adeo ut bona sua cum his aequis partibus diuiserit quia illi bello ciuili erant spoliati: «Le chevalier romain Proculeius montra une si grande affection envers ses frères, Scipion et Muréna, qu'il partagea avec eux ses propres biens vu que ceux-ci avaient été dépouillés par la guerre civile ». Proculeius avait rempli pour Auguste des missions délicates (PLIN., H.N. 7, 148; DC., 51, 11, 4; PLUT., Ant. 78-79). Auguste aurait même songé à faire de lui son gendre (TAC., An. 4, 40,6). Selon R. SYME 1984 : 1089, il pourrait exister une parenté par alliance entre Sallustius Crispus et Proculeius, ce qui expliquerait en partie l'introduction du second dans une ode dédiée au premier (le nomen Proculeius apparaît dans des inscriptions d'Amiterne, patria de l'historien Salluste).

18. SAL., C. 11.

19. La première ambassade de Crinagoras auprès d'Octave a eu lieu en 26-25. On situe le deuxième recueil des Odes entre 30 et 23 . Rien n'interdit donc une rédaction quasi contemporaine des deux poèmes.

20. ALEXANDER 1943 : pp. 192-201; CALDER $1961: 175$.

21. La sœur de Pison, Calpurnia, avait été la femme de Jules César, premier lien avec la famille impériale. Si Pison le Pontife n'a pas été lui-même le conjoint d'une princesse, en digne héritier de la prudence politique paternelle (syme 1986: p. 331), sa fille a été mariée avec L. Nonius Asprenas, dont le père, ami personnel d'Auguste, avait épousé une des soeurs de P. Quinctilius Varus (une autre étant mariée à Sextus Apuleius, fils d'Octavie, demi-soeur d'Auguste). 
22. L'épître pourrait dater de 15 av. J.C. (elle honorerait le consulat de Pison) ou de 10-9 av. J.C. (elle célèbrerait les supplicationes et les ornamenta triumphalia décernés par le Sénat à Pison pour ses succès militaires en Thrace pendant les années 12-10, [DC., 54, 34, 7]). Pendant sa campagne militaire, Pison, legatus Augusti aurait été investi de secreta mandata par le Prince (sÉN., Ep. 83, 14). L'identification du destinataire d'Horace et du Pontifex a été faite par Porphyrion suivi par SYME $1986: 379$ et GRIMAL $1968: 28$.

23. Anth. 9, 25: «Phébus, gardien du port des Céphalléniens, habitant de la plage de Panorme en face de la rocailleuse Ithaque, permets que je puisse voguer vers la terre d'Asie, à travers des flots propices à la navigation, dans le sillage du long navire de Pison; et fais en sorte que mon vaillant souverain lui soit favorable, et soit favorable à mes chants » (Traduction personnelle). Pison fut proconsul en Asie en 8 av. J.C.; il est ici transformé en nouvel Ulysse.

Anth. 6, 241: «Casque, je jouis d'un double privilège: ma vue est un sujet de joie pour les amis et de crainte pour les ennemis. J'appartenais autrefois à Pylaiménès; maintenant je suis à Pison. Un tel casque ne pouvait convenir à d'autres cheveux ni une autre chevelure à ce casque. » Le nom de Pylaiménès permet astucieusement de louer à la fois l'érudition littéraire de Pison (c'est le nom que porte dans l'Iliade le chef des Paphlagoniens dont les soldats, après la chute de Troie, auraient abordé en Thrace avant de s'installer en Vénétie) et ses fonctions de légat consulaire en Galatie-Pamphylie (Pylaiménès est le fils d'Amyntas, le roi de Galatie créé par Antoine et confirmé par Auguste).

Anth.6, 249: «Ce cierge revêtu d'une tunique de cire, ce flambeau fumeux de Cronos, fait de jonc tressé et de léger papyrus, Antipater l'offre à Pison en hommage. S'il m'allume et adresse aux dieux une prière, l'éclat dont je brillerai montrera qu'ils ont entendu ses voeux. » Ce cadeau est celui du client pauvre à son patron le jour des Saturnales (MACR., 1, 7, 31-33 et 1, 1, 47-49). Cette mince offrande, tenuis et humilis, symbolise sans doute la poésie légère prisée par Pison et Antipater.

Anth. 6, 335: «Chapeau de feutre, autrefois le couvre-chef commode des Macédoniens, abri en temps de neige, casque pendant la guerre, j'ai eu soif de boire ta sueur, brave Pison et je suis venu d'Emathie pour orner tes tempes ausoniennes. Reçois-moi en ami: bientôt cette même coiffure qui jadis mit les Parthes en fuite rangera encore les Thraces sous ta domination ».

Anth. 9, 541: "Theogène nous envoie à Pison, coupes artistiquement travaillées; nous contenons tout le ciel à nous deux. Car nous sommes faites ensemble d'une sphère coupée et, sur l'une de nous, se voient les constellations du sud, sur l'autre, celles de Borée. Mais toi, sur Aratos, ne jette plus les yeux et, en nous vidant chacune une fois, regarde tous les Phénomènes." L'épigrammatiste cite sans doute le mathematicus Theogène d'Alexandrie (suÉT., Aug. 94). Son cadeau, qui rappelle la coupe à sujet astronomique de Virgile (B. 3, 36), correspond particulièrement à ses activités d'astrologue.

24. Anth. 9, 19: «Coupe, aujourd'hui, Gaius, la première douce moisson de tes joues et les jeunes boucles de ton menton; ton père Lucius recevra dans sa main le duvet désiré de cette barbe qui grandit pour voir de nombreux soleils. On offre des présents dorés; moi j'offre de joyeux distiques; car, en vérité, la Muse n'est pas inférieure à la richesse » (traduction personnelle). Le jeune homme en question serait l'aîné des deux fils qu'Horace décrit comme encore modelé par les paroles paternelles (A. P. 366-367). Cela implique donc une datation tardive de l'Art poétique (11-8 av. J.C: les lauriers de Thrace et le gouvernement en Syrie). Mais il n'y a aucune trace historique du second fils mentionné par Horace.

25. Les liens entre Philodème et Siron étant attestés, les relations entre Horace et Pison le Pontife se seraient tissées dans les milieux épicuriens de Campanie.

26. Cf. ses poèmes en l'honneur d'Euphorion, de Rhianos et de Parthénios et sa décision de placer leurs portraits et leurs oeuvres inter ueteres et praecipuos auctores (sUÉT., Tib. 70). Ce philhellénisme n'était pas le seul point commun entre Pison et Tibère: tous deux partageaient un amour immodéré de la boisson (sUÉT., Tib. 42,1). 
27. SÉN., Ep. 83, 14 et VELL., 2, 98, 1.

28. SUÉT., Tib. 42, 1.

29. VOISIN 2010.

30. GRIMAL 1968

31. HOR., S. 1, 10, 36-37 et 2, 5, 4 à rapprocher de P. 17.

32. DUPONT $1985: 335-341$.

33. La définition qu'Horace donne du poète idéal, à la fois possesseur des techniques de l'écriture et détenteur de la sagesse philosophique des droits et des devoirs qui régissent la société romaine, rappelle la définition de l'orateur idéal selon Cicéron. Devons-nous reconnaître une autre allusion à Cicéron? De même que l'orateur avait vilipendé Piso Caesoninus, l'épicurien et le politicien débauché, Horace aurait choisi ses héritiers comme exemple d'un goût poétique dépravé.

34. C'est le sens du verbe sapere (GRIMAL $1986: 6$ ).

35. Point de vue défendu par LAUGIER 1990 : pp. 159-166. Il existe une troisième solution (FRISCHER 1991 : 52-68): le père est Piso Caesoninus lui-même. Elle nous semble contestable, dans la mesure où elle n'identifie qu'un seul des fils, l'aîné (consul en 15 av. J.C.). De plus, l'âge de ce dernier ne convient guère au contenu des vers 366-367, même en supposant une datation haute de l'Art Poétique.

36. TAC., An. 1, 74, 5.

37. TAC., An. 2, 43.

38. TAC., An. 2, 34 et 3, 12, 2.

39. Ce ralliement valut plus tard aux deux fils des épouses de choix: l'aîné épousa une Munatia Plautina, la petite-fille de Munatius Plancus; le second une Statilia, fille du maréchal d'empire Statilius Taurus (sYME $1986: 369-377$ ).

40. Horace cherche une synthèse entre le mos maiorum et les charmes de l'hellénisme (MICHEL 1988 : 199-209)

41. Autrefois chargé par Pompée de sélectionner les pièces qui devaient être jouées à l'inauguration de son théâtre, il avait choisi la Clytemnestre d'Accius et le Cheval de Troie de Livius Andronicus. L'une et l'autre tragédies permettaient des mises en scène somptueuses où le butin pris sur les peuples d'Asie figurait en bonne place. Le peuple fut satisfait, Cicéron le fut moins ( CIC., Fam. 7, 1, 1).

42. Cf. l'autoportrait d'Horace aux vers 306-308: munus et officium, nil scribens ipse, docebo,/ unde parentur opes, qui alat formetque poetam,/ quid deceat, quid non, quo uirtus, quo ferat error. L'accumulation et le rythme de plus en plus alerte des interrogatives indirectes, l'opposition absurde entre nil scribens ipse et docebo révèlent qu'Horace ne prend pas complètement au sérieux son rôle de professeur. L'Art poétique ne serait-il pas (aussi) une parodie des traités à la mode? Nous pensons, par exemple au De Vrbanitate de Domitius Marsus. Si l'on prend l'expression nil scribens ipse au sens propre, il faut comprendre, qu'Horace n'écrit pas de pièces de théâtre ( GRIMAL $1986: 67-80$ ).

43. Strabon a sans doute été en relation avec $\mathrm{Cn}$. Pison, le consul de 7 av. J.C. dont il cite une description de l'Afrique et qui lui a fourni des informations de première main sur la province consulaire de Tarraconaise (syme 1986 : 370-371). Ce lien d'un consulaire avec un géographe grec ne suffit pas pour faire de $\mathrm{Cn}$. Pison un amateur de littérature. D'ailleurs, Strabon, dans sa préface, indique que son ouvrage s'adresse à des généraux et à des hommes d'État.

44. Plus précisément, de la seconde partie de l'année, puisque Sestius fut nommé consul suffect lorsque Auguste quitta sa charge de consul le $1^{\text {er }}$ juillet.

45. Pourtant, Sestius, comme de nombreux épicuriens de l'aristocratie, accepta des charges politiques: outre son consulat, un commandement de légat consulaire en Espagne Ultérieure ( PLIN., H.N. 4, 111). 
46. Les réflexions générales $d$ 'Horace sur le poème et le poète dans les première et troisième parties de l'Art poétique s'appliquent à Horace lui-même. Lorsque l'écrivain insiste sur le decorum, sur la brièveté, sur la douceur des carmina et sur leur utilité dans l'ordre de la pietas et de la uirtus politique, il esquisse un programme qui correspond exactement aux résultats obtenus dans les Odes (MICHEL 1988 : pp. 204).

47. En 43, Octave célèbre pour sa mère des funérailles nationales (DC., 47, 17, 6 ; suÉT., Aug. 61, 2). Plus grave pour les survivants de l'aristocratie républicaine, de telles funérailles sont organisées en l'honneur de l'affranchi Sphaerus, son pédagogue (DC., 48, 33, 1). Il existe cependant un précédent : en 54 av. J.C., à la demande du peuple, Pompée célébra des funérailles nationales sur le champ de Mars pour son épouse Julia. En commémoration de la victoire de 36, est décrété un banquet annuel dans le temple de Jupiter Capitolin, auquel assistent l'épouse et les enfants d'Octave (DC., 49, 15, 1). Après les premiers succès militaires en Dalmatie, des statues de Livie et d'Octavie sont dressées et leurs personnes sont déclarées sacro-saintes. Enfin, les effigies des membres de la famille du Prince, y compris les femmes et les jeunes enfants, attirent tous les regards sur le Mausolée du Champ de Mars et sur l'Ara Pacis. Ces représentations officielles ont connu une grande popularité. En témoigne l'attitude de la classe moyenne et des affranchis qui imitent les portraits de l'idéologie officielle et privilégient, sur leur tombeaux, la représentation des groupes familiaux et des enfants (KLEINER 1984 : 107-135).

48. En 42 , l'épouse de Tiberius Nero, rallié aux républicains, donnait naissance à Tibère.

49. Il est difficile d'y voir une distinction honorifique indépendante de toute réalité concrète ( WILLIAMS 1969 : p. 99). Le critique met en parallèle cette expression avec la distinction du ius trium liberum décernée à Livie par le sénat en 9 av. J.C. Je pense que, si le titre de uniuira ne fut jamais officiellement accordé à Livie, c'est qu'il aurait fâcheusement rappelé les circonstances du mariage de Livie et d'Octave.

50. ALFONSI 1976: 288-294. Les fragments concernant Aitia ont la forme d'une épitaphe traditionnelle mais exaltent la nature divine de son fils et la louent d'avoir donné naissance à un dieu (DURET 1983 : 1484-1486).

51. CRINAGORAS, Anth. 9, 239 pour accompagner un envoi de vers lyriques à Antonia; 6, 244, sur l'accouchement d'Antonia. Athénodore, le philosophe stoïcien attaché à Octave, adressa une consolation à Octavie (PLUT. Publ. 17), sans doute après la mort de son mari en 40. De même, Aréios d'Alexandrie écrivit une consolation pour Livie, après la mort de Drusus.

52. Tibulle célèbre ainsi, avec une touchante fausse naïveté, l'anniversaire de Cornutus qui vient de se marier $(2,2)$, et fait une allusion malicieuse au mariage de Titius $(1,4,73-74)$.

53. CATUL., $45 ; 61$.

54. GROSS 1984 : pp. 42-50.

55. En 4 ap. J.C., Auguste déclare qu'il adopte Tibère pour le salut de l'Etat (sUÉT., Tib. 21, 3).

56. Ainsi, Auguste a démenti formellement, après sa maladie de 23, avoir désigné Marcellus comme son successeur et, pourtant, le peuple était certain de cette désignation (DC., 58, 30-31). Marcellus était praeparatus successioni (sÉN., Polyb. 15, 3), ce qui conférait d'emblée la succession ( DC., 54, 18, 1).

57. Il a distribué de l'argent au peuple au nom de Marcellus et ce dernier montait un des chevaux de son chariot triomphal (DC., 51, 21, 3 ; sUÉT., Tib. 6, 4); en 24 av. J.C., il annonce que Marcellus va être consul alors que ce dernier n'aura que 22 ans. Gaius et Lucius apparaissent sur les monnaies dès 13 av. J.C. En 6-5 av. J.C., un serment provenant de Bétique, donc d'une région peu familiarisée avec les traditions monarchiques orientales, est prêté à César Auguste, à ses enfants et à ses descendants (GONZALES $1988: 113-127)$.

58. CRINAGORAS, Anth. 6, 161 rend hommage à la guerre d'occident, c'est-à-dire à la première expédition militaire de Marcellus contre les Cantabres en 25 av. J.C. à l'âge de 18 ans; elle célèbre également la première barbe de Marcellus, symbole privé de son passage à l'âge adulte; 9, 545 
accompagne l'envoi d'un exemplaire de l'Hécalè et glorifie la vigueur et la gloire futures du jeune homme comparé à Thésée. En revanche, Octavie, en deuil de son fils, refusa nombre de poèmes en l'honneur de Marcellus (sÉN., Marc. 2, 5).

59. VoIsin 2010 : pp. .

60. Si l'intérêt politique l'avait emporté, Horace aurait dû célébrer également Paulus Aemilius Lepidus (consul suffect en 34 av. J.C.), époux de Cornélie, la demi-soeur de Julie; P. Cornelius Scipio, frère de Cornélie et consul en 16 av. J.C.; Sextus Appuleius, le consul de 29, dont le père avait épousé la demi-soeur d'Auguste, Octavie; son frère, Marcus Appuleius, consul en 20 av. J.C.; L. Domitius Ahenobarbus, consul en 16 av. J.C. et mari d'Antonia l'aînée; M. Messalla Appianus, consul en 12 av. J.C. et mari de Marcella la jeune; P. Quinctilius Varus, consul en 13 av. J.C. et gendre d'Agrippa.

61. PLIN., Ep. 5, 3.

62. NÉRAUDAU 1979 : 368-373.

63. Si l'on excepte l'enfant sans nom de la quatrième Bucolique virgilienne. Là encore, le thème est traité à l'avance, avant que l'idéologie officielle ne s'en empare. De plus, le texte transcende à ce point tout événement particulier qu'aucun lecteur ancien ou moderne n'a pu identifier cet enfant avec certitude ni même décider s'il s'agit d'un enfant bien particulier ou d'un symbole BEAUJEU 1982 : 186-215.

\section{RÉSUMÉS}

Cet article se propose d'étudier les liens éventuels unissant Horace et les poètes grecs contemporains. Une première analyse compare deux poèmes adressés à Caius Sallustius Crispus, le neveu du fameux historien, Salluste: l'un est de Crinagoras, l'autre d'Horace. A l'hommage courtisan que l'épigrammatiste grec adresse à l'ami du Prince, le poète latin préfère un portrait ambigu, destiné moins à Sallustius qu'à des lecteurs lettrés, amusés par la comparaison entre l'historien moraliste pastiché par le poète et le politique opportuniste. Ensuite, de manière plus générale, est présentée une évaluation critique des mentions que fait le lyrique des personnalités célébrées par Crinagoras, Antipater de Thessalonique ou Apollonide de Nicée. Dans le choix de ses dédicataires, Horace ne vise pas un intérêt immédiat et courtisan ; il s'adresse avant tout à un lectorat cultivé, sub specie aeternitatis. Ainsi, conformément à la tradition littéraire romaine et fidèle à son projet poétique original, Horace s'est interdit d'exploiter, dans les Odes, le thème, si cher à Auguste, de la famille impériale, se distinguant, là encore, des poètes grecs courtisans.

This article proposes to study the possible links joining Horatius and the Greek contemporaneous poets. A first analysis compares two poems adressed to Caius Sallustius Crispus, the nephew of the famous historian, Sallustius : the first is by Crinagoras, the second by Horatius. To the sycophantic tribute that the Greek epigrammatist adresses to the Prince's friend, the Roman poet prefers an ambiguous portrait less intended to Sallustius Crispus than to a well-read public, amused by the comparison between the moralist historian imitated by the poet and the opportunistic politician. Then, in a more general way, a critical evaluation is presented, an assessment of the mentions that makes the lyric poet of the personalities celebrated by Crinagoras, Antipater of Thessaloniki or Apollonides of Nicaea. In the choice of his dedicatees, Horatius does not aim at an immediate and sycophantic interest; principally, he speaks to a cultured public, sub specie aeternitatis. So, in conformity with the literary Roman tradition and 
true to his original poetic plan, Horatius has refrained, in his Odes, from exploiting the theme of the imperial family, so dear to Augustus, and so distinguishing himself, here again, from the Greek court poets.

INDEX

Mots-clés : épigrammatistes grecs, Horace, poésie de cour, poésie lyrique augustéenne

Keywords : Court poetry, Greek epigrammatists, Horace, Lyric augustean poetry

\section{AUTEUR}

\section{DOMINIQUE VOISIN}

Maître de Conférence de latin. CTEL Université de Nice Sophia Antipolis Ses domaines de recherche sont : Les relations dans le monde des lettres au siècle d'Auguste Poétique de la réécriture et transgénéricité - Poésie néo-latine. 